\title{
BMJ Open Instruments for assessing the preferences for everyday living of older people with various care needs: protocol for an evidence map
}

\author{
Mike Rommerskirch-Manietta (D) ,, ${ }^{1,2}$ Daniel Purwins, ${ }^{1,2}$ Kimberly Van Haitsma, ${ }^{3}$ \\ Katherine Abbott, ${ }^{4,5}$ Martina Roes (i) ${ }^{1,2}$
}

To cite: RommerskirchManietta M, Purwins D, Van Haitsma K, et al. Instruments for assessing the preferences for everyday living of older people with various care needs: protocol for an evidence map. BMJ Open 2021;11:e048921. doi:10.1136/ bmjopen-2021-048921

- Prepublication history and additional supplemental material for this paper are available online. To view these files, please visit the journal online (http://dx.doi.org/10.1136/ bmjopen-2021-048921).

Received 11 January 2021 Accepted 23 August 2021

Check for updates

(C) Author(s) (or their employer(s)) 2021. Re-use permitted under CC BY-NC. No commercial re-use. See rights and permissions. Published by BMJ.

For numbered affiliations see end of article.

Correspondence to Mr Mike RommerskirchManietta;

Mike.Rommerskirch-Manietta@ dzne.de

\section{ABSTRACT}

Introduction Consideration of the preferences for everyday living of older people with various care needs is a prerequisite for person-centred and evidencebased nursing care. Knowledge of and respect for these preferences by nursing staff are associated with better care outcomes for older people with various care needs. To assess preferences in a structured way, instruments focusing on different topics of everyday living appear to be useful. It is unclear which instruments exist for assessing preferences for everyday living. The aim of this planned review is to identify relevant instruments for assessing the preferences for everyday living of older people with various care needs in the form of an evidence map. Additionally, gaps requiring further research will be presented.

Methods and analysis To identify the different instruments, we will conduct a systematic search in the electronic databases MEDLINE (via PubMed), CINAHL and PsycINFO (via EBSCO). In addition, we will perform backward and forward citation tracking via reference lists and Google Scholar. The identified records will be independently screened (title/abstract and full text) by two reviewers. Data from the included studies will be extracted independently by the same two reviewers. In all three steps, the results will be checked for deviations, and if there are any deviations, they will be discussed. If no consensus can be achieved through discussion, a third reviewer will be engaged. All study designs will be included, and there will be no limitations regarding the publication status or time period. We will include all studies published in English and German that use instruments focusing on the assessment of preferences for everyday living in people older than 60 years of age with various care needs. For data charting, we will extract the number, categories and types of preferences, the care setting for which the instrument was developed and, if available, psychometric properties. Finally, the various extracted results will be presented in the form of tables and a bubble plot.

Ethics and dissemination There are no ethical concerns related to the construction of an evidence map, and ethical approval was given by the Witten/Herdecke University (application number 226/2020). We will discuss our results with practitioners in the field of nursing care and persons with various care needs. We will also make our results available to practitioners in an upcoming Project (PELI-D II)

\section{Strengths and limitations of this study}

- The evidence map will be, for the first time, identifying instruments used for assessing preferences for everyday living.

- We will include all study designs with no time period or publication status restrictions.

- We will use a broad approach of the term everyday living to identify a high variety of different instruments.

- We may miss instruments published outside of journals (eg, grey literature).

- Main limitation will be the current lack of a general definition for everyday living in the context of preferences.

and to the public at (inter)national conferences and in the form of practice and peer-reviewed articles.

\section{INTRODUCTION}

The focus of care for older people with various care needs is shifting from a medicalcentred to a person-centred approach across care settings. ${ }^{1}$ A person-centred approach is characterised by the way nursing staff tailor their services to wrap around the individual needs of clients or residents. ${ }^{2-4}$ In addition, there is a demand for nursing staff to provide interventions that are evidence based. ${ }^{5}$ For person-centred and evidence-based nursing care, it is a prerequisite that nursing staff have knowledge of the preferences of their clients or residents. This knowledge enables nursing staff to adapt their care interventions based on the preferences and individualised needs of the older people in their care. ${ }^{67}$ This corresponds to a person-centred care approach that affirms the individuality and preferences of clients or residents. ${ }^{78}$ In addition, the preferences identified during interactions between nursing staff and client or resident can contribute to the development of internal 
evidence and thus promote evidence-based nursing care. ${ }^{6}$ In both of these nursing care approaches, information about the preferences for everyday living of older persons with various care needs appears to be a key aspect. This is because one core aspect of nursing is to support older people with various care needs in completing their activities of daily living. ${ }^{9-11}$ Furthermore, studies have shown that taking into account preferences for everyday living can lead to an improvement in nursing care outcomes, such as continence or nutrition status of people with various care needs. $^{12} 13$

In the assessment of preferences for everyday living, structured instruments appear to be useful. ${ }^{14}$ To date, there is no systematic overview of the various instruments and their purposes. As a result, practitioners and researchers are not able to determine which instruments can be used in their care setting or what scientific gaps currently exist.

In this article, we report the protocol for our evidence map. It follows the guidelines for systematically identifying and reporting the types and characteristics of instruments used for assessing the preferences for everyday living of older people who receive long-term services and supports in a variety of settings.

\section{METHODS AND ANALYSIS}

The evidence map is a newer review method for systematically processing research findings. ${ }^{15}$ For an evidence map, it is essential that a broad research question be systematically investigated, and the results are presented by visual mapping of the identified evidence and descriptions of evidence gaps. ${ }^{16}$ In contrast to systematic reviews, as defined by the Cochrane Collaboration, ${ }^{17}$ for evidence maps, it is not necessary to assess the quality or analyse the strength of evidence of the included studies, since the purpose of evidence maps is not to provide recommendations for interventions. In addition, in contrast to a scoping review, as defined by the Joanna Briggs Institute, ${ }^{18}$ the focus of an evidence map is on the descriptive visual presentation of the current research landscape, which enables practitioners and researchers to obtain information on existing evidence and research gaps for a specific topic in a user-friendly visual way. 15161819

For our planned evidence map, starting in November 2020 and scheduled to end in August 2021, we follow the iterative framework for scoping studies developed by Arksey and $\mathrm{O}^{\prime}$ Malley ${ }^{20}$ which was further modified by Levac et al. ${ }^{19}$ This framework contains six different stages: (1) identifying the research question, (2) identifying relevant studies, (3) study selection, (4) charting the data, (5) collating, summarising and reporting the results and (6) consulting. In addition, we followed, whenever applicable, the Preferred Reporting Items for Systematic Review and Meta-analysis Protocols guidelines ${ }^{21}$ for reporting this protocol (online supplemental table 1).
Stage 1: identifying the research question

In the context of a pilot study focusing on the translation and psychometric testing of an instrument for assessing the preferences for everyday living of older people in various care settings, ${ }^{1422-24}$ discussions among the research team resulted in several questions regarding further development of the instrument. In this context, how other instruments are structured and which topics they assess was discussed. This led to an explorative literature search to identify a systematic overview of currently available instruments for this topic. However, no systematic overview could be identified. Based on this, we defined the following research question for our evidence map: 'Which instruments exist for assessing the preferences for everyday living of older people with various care needs?'

\section{Stage 2: identifying relevant studies}

In November 2020, we will conduct a literature search to identify relevant instruments for assessing preferences for everyday living in the following electronic databases: MEDLINE (via PubMed), CINAHL and PsycINFO (via EBSCO). Our search string was developed for MEDLINE (via PubMed) (online supplemental table 2) and adapted for the other two databases by a single reviewer (MR-M) according to RefHunter V.5.0. ${ }^{25}$ Additionally, we will provide backward and forward citation tracking (via reference lists and Google Scholar). For included studies that do not report all characteristics of interest for the instrument, we will identify additional publications with information about, for example, the development and psychometric testing of the instrument.

\section{Stage 3: study selection}

Records identified through our electronic database search will be imported into Covidence ${ }^{26}$ and automatically checked for duplicates. The titles, abstracts and full texts of identified records will be screened independently by two reviewers (MR-M/DP) in Covidence against the eligibility criteria (table 1 ). The results of these two screening processes will be discussed between the two reviewers in the case of different ratings. If no consensus can be reached by discussion, a third reviewer (MR) will be involved. The first 200 records screened will be used to test our eligibility criteria and adjust them if necessary. An adjustment will be necessary if the variation in ratings (include/exclude) between the two reviewers is more than $20 \%$. If this is the case, we will further refine our eligibility criteria through a team discussion and report the changes in articles published about the evidence map.

\section{Stage 4: charting the data}

For data charting, we will create a form in Windows Excel (table 2). Two reviewers (MR-M/DP) will independently extract the data from the included studies. After finishing this extraction process, the same two 
Table 1 Eligibility criteria

\section{Criteria Definition}

Population We will include studies with a study population of people older than 60 years with various care needs. Because care dependency is defined variously in the international context, people with few care needs or a need for day-structuring activities only are not always included as people with care needs, especially in studies that focus on non-traditional care settings such as adult day service. ${ }^{28-30}$ We will exclude studies that focus on people receiving palliative care.

Concept of We will consider only studies that used instruments that focus on assessing preferences for everyday living. interest In this context, instruments are also considered relevant when more then one of the items are related to the assessment of preferences for everyday living. We understand everyday living as a daily routine in which decisions on different topics of life are made by each person based on individual preferences. Following the conceptual nursing model I based on activities of living developed by Roper et al, ${ }^{10}$ we will consider the following to be preferred topics:

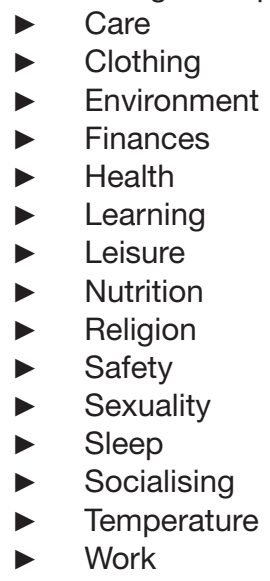

Study design Because the objective is to identify instruments for assessing preferences for everyday living and the broad ways in which the instruments are used in research, we will consider all types of study designs.

Others We will only include studies published in English and German. There will be no restrictions on publication status or date.

reviewers will check every item of the extracted data for consistency. If there are any differences in the extractions, they will be discussed. If no consensus can be reached, a third reviewer (MR) will be involved. Furthermore, we will test the data charting form for the first five included studies. If there is a $20 \%$ difference between the extracted data of the two reviewers, we will revise the data charting form on team discussion and report the changes in articles published about the evidence map.

\begin{tabular}{ll}
\hline Table 2 Data charting framework \\
\hline Domains
\end{tabular}




\section{Stage 5: collating, summarising and reporting the results}

The extracted data (table 2) will be reported via tables and a bubble plot. We will report general aspects of the identified instruments (eg, developer details) in the form of tables. The more specific information about the instruments will be visualised in the form of a bubble plot. For this purpose, we will use R V.4.0.3 and ggplot2 to collate, summarise and graph the identified instruments according to the type of preferences, the population and the care settings for which each instrument was developed. ${ }^{27}$ Therefore, one bubble will be created, in the bubble plot, for each included instrument in the evidence map. To illustrate the number and categories of the different preferences (eg, leisure or care) of the included instruments, we will modify the size and colour of the different bubbles accordingly. For example, the size of the bubble will be based on the number of preferences (eg, questions for or visuals of preferences) that an instrument contains. Additionally, for information about the assessment method (eg, using a sorting approach or questionnaire), aim (eg, research or practice) and psychometric properties (eg, reliability and validity) of the instrument, we will add an extra visual element around every mapped bubble, such as a symbol (eg, plus or minus). The aim is by graphically representing the identified instruments in form of bubbles, a quick overview is available for practitioners and/or researchers about possible instruments for example their care setting or the preferences of interest.

\section{Stage 6: consultation}

Since stage 6 is described as being in parallel to the other stages, ${ }^{20}$ we will discuss our results with a minimum of one practitioner from the field of nursing care after completing stages 3, 4 and 5. Practitioners will be recruited from partner nursing facilities of the German Center for Neurodegenerative Diseases (DZNE). Additionally, we will also involve a minimum of one person from the patient advisory board of the DZNE and/or a person who received care from a partner nursing facility in the discussion. The first aim is to discuss the graphically design of the bubble plot to ensure ease of use and accessibility. Second we will discuss the identified instruments according to their use in practice and to obtain an impression of how the instruments reflect the preferences of older people with various care needs. Results will be influence the design of the bubble plots and will be presented in the result and discussion part of the upcoming publication. In addition, a discussion among the research team members will take place after each stage to evaluate the current approach, the results and the need for further adjustments of the procedure.

\section{Patient and public involvement}

We will involve a minimum of one practitioner from the field of nursing care and a minimum of one person from the patient advisory board of the DZNE and/or persons who have visit an adult day service on a regular basis.
The involvement is planned for stage six consultation to discuss the results of our evidence map.

\section{Ethics and dissemination}

For our review, there are no ethical concerns. An ethical proposal for a research project focusing on understanding and assessing the preferences for leisure activities of people with care needs in adult day services (PELI-D II), including the evidence map, has been submitted to the Witten/Herdecke University and has received ethical clearing (application number 226/2020). We will present our results to practitioners in participating facilities of the upcoming PELI-D II Project and at (inter)national conferences and publish them in journals for practitioners and peer-reviewed journals. In addition, we will address possible gaps in the current research landscape identify by the evidence map (eg, missing preference instruments for people living with dementia) and incorporate them into possible future projects.

\section{Author affiliations}

${ }^{1}$ German Center for Neurodegenerative Diseases Witten (DZNE), Witten, Germany ${ }^{2}$ Department of Nursing Science, Faculty of Health, Witten/Herdecke University, Witten, Germany

${ }^{3}$ College of Nursing, Pennsylvania State University, Pennsylvania, Pennsylvania, USA ${ }^{4}$ Department of Sociology and Gerontology, Miami University, Oxford, Ohio, USA

${ }^{5}$ Scripps Gerontology Center, Oxford, Ohio, USA

\section{Twitter Mike Rommerskirch-Manietta @ rochmro}

Contributors MR-M wrote the initial draft of the protocol. DP, KVH, KA and MR revised the manuscript. MR and DP assisted in the identification of the electronic databases and reviewed the keywords and the search string. KVH, KA and MR are supervisors of this project. All authors read and approved the final manuscript.

Funding The authors have not declared a specific grant for this research from any funding agency in the public, commercial or not-for-profit sectors.

Competing interests None declared.

Patient consent for publication Not required.

Provenance and peer review Not commissioned; externally peer reviewed.

Supplemental material This content has been supplied by the author(s). It has not been vetted by BMJ Publishing Group Limited (BMJ) and may not have been peer-reviewed. Any opinions or recommendations discussed are solely those of the author(s) and are not endorsed by BMJ. BMJ disclaims all liability and responsibility arising from any reliance placed on the content. Where the content includes any translated material, BMJ does not warrant the accuracy and reliability of the translations (including but not limited to local regulations, clinical guidelines, terminology, drug names and drug dosages), and is not responsible for any error and/or omissions arising from translation and adaptation or otherwise.

Open access This is an open access article distributed in accordance with the Creative Commons Attribution Non Commercial (CC BY-NC 4.0) license, which permits others to distribute, remix, adapt, build upon this work non-commercially, and license their derivative works on different terms, provided the original work is properly cited, appropriate credit is given, any changes made indicated, and the use is non-commercial. See: http://creativecommons.org/licenses/by-nc/4.0/.

ORCID iDs

Mike Rommerskirch-Manietta http://orcid.org/0000-0002-1533-3006

Martina Roes http://orcid.org/0000-0003-4531-8584

\section{REFERENCES}

1 Epstein RM, Street RL. The values and value of patient-centered care. Ann Fam Med 2011;9:100-3.

2 McCormack B, Roberts T, Meyer J, et al. Appreciating the 'person' in long-term care. Int J Older People Nurs 2012;7:284-94. 
3 White DL, Newton-Curtis L, Lyons KS. Development and initial testing of a measure of person-directed care. Gerontologist 2008;48 Spec No 1:114-23.

4 American Geriatrics Society Expert Panel on Person-Centered Care. Person-Centered care: a definition and essential elements. J Am Geriatr Soc 2016;64:15-18.

5 WHO. European strategic directions for strengthening nursing and midwifery towards health 2020. Copenhagen: World Health Organization Regional Office for Europe, 2015.

6 Behrens L, Langer G. Evidence-based nursing caring - methoden und ethik der pflegepraxis und versorgungsforschung. Bern: Hans Huber, 2010.

7 McCormack B, McCance TV. Development of a framework for person-centred nursing. J Adv Nurs 2006;56:472-9.

8 McCormack B, McCance TV. Person-centered nursing - theory and practice. Oxford/lowa: Wiley-Blackwell, 2010.

9 Bartholomeyczik S. Prävention und gesundheitsförderung ALS Konzepte Der Pflege. Pflege \& Gesellschaft 2006;11:210-23.

10 Roper N, Logan WW, Tierney AL. The roper-logan-tierney model of nursing: based on activities of living Edingburgh. Amsterdam: Elsevier Health Sciences, 2000.

11 Orem DE. Nursing: concepts of practise. New York: McGraw-Hill, 1985.

12 Simmons SF, Schnelle JF. Individualized feeding assistance care for nursing home residents: staffing requirements to implement two interventions. J Gerontol A Biol Sci Med Sci 2004;59:M966-73.

13 Thompson DL, Smith DA. Continence restoration in the cognitively impaired adult. Geriatr Nurs 1998;19:87-90.

14 Rommerskirch-Manietta M, Roes M, Stacke Tl, et al. Präferenzen von Menschen mit Pflegebedarf - Eine explorative Analyse von Pflegedokumentationen in verschiedenen Settings. HeilberufeScience 2021;12:13-21.

15 Schmucker C, Motschall E, Antes G, et al. [Methods of evidence mapping. A systematic review]. Bundesgesundheitsblatt Gesundheitsforschung Gesundheitsschutz 2013;56:1390-7.

16 Miake-Lye IM, Hempel S, Shanman R, et al. What is an evidence MAP? A systematic review of published evidence maps and their definitions, methods, and products. Syst Rev 2016;5:28

17 Higgins PJ, Green S. Cochrane handbook for systematic reviews of interventions - cochrane book series. The Atrium, Southern Gate,
Chichester, West Sussex, England.: The Cochrane Collaboration and John Wiley \& Sons Ltd, 2008.

18 The Joanna Briggs Institute. Joanna Briggs Institute reviewers' manual: 2015 edition/Supplement. Australia: The Joanna Briggs Institute, 2015.

19 Levac D, Colquhoun H, O'Brien KK. Scoping studies: advancing the methodology. Implementation Science 2010;5:1-9.

20 Arksey H, O'Malley L. Scoping studies: towards a methodological framework. Int J Soc Res Methodol 2005;8:19-32.

21 Shamseer L, Moher D, Clarke M, et al. Preferred reporting items for systematic review and meta-analysis protocols (PRISMA-P) 2015: elaboration and explanation. BMJ 2015;350:97647.

22 Stacke TI, Bergmann JM, Ströbel AM, et al. Preferences for everyday living inventory (peli): study protocol for piloting a culture-sensitive and setting-specific translated instrument in German care settings (PELI-D). BMJ Open 2020;10:e030268.

23 Rommerskirch-Manietta M, Roes M, Palm R, et al. Präferenzen des alltäglichen Lebens in Der Pflegedokumentation - eine Dokumentenanalyse in verschiedenen pflegerischen settings. Pflege 2021;34:191-202.

24 Stacke TI, Manietta C, Purwins D, et al. Übersetzung und kultursensitive Anpassung des preferences for everyday living Inventory $\odot$ für pflegerische Versorgungssettings. Pflege 2021:1-8.

25 Nordhausen T, Hirt J. Manual zur literaturrecherche in fachdatenbanken - RefHunter. Halle: Martin-Luther-Universität HalleWittenberg \& Ostschweizer Fachhochschule, 2020.

26 Covidence. Systematic review software. secondary systematic review software, 2020. Available: www.covidence.org

27 Computing R. A language and environment for statistical computing. Vienna: R Core Team, 2013.

28 Hedayati M, Sum S, Hosseini SR, et al. Investigating the effect of physical games on the memory and attention of the elderly in adult day-care centers in Babol and Amol. Clin Interv Aging 2019;14:859-69.

29 Hweidi IM, Gharaibeh BA, Al-Obeisat SM. Adult day care services: directions for moving forward. Int J Gerontol 2018.

30 Schmitt EM, Sands LP, Weiss S, et al. Adult day health center participation and health-related quality of life. Gerontologist 2010;50:531-40. 